\title{
Femicidio y Overkill: A Propósito de un caso.
}

\section{Femicide and Overkill: A case report.}

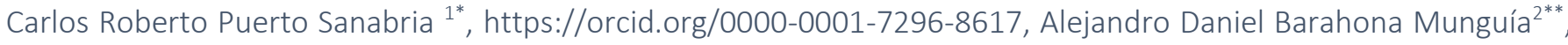
https://orcid.org/0000-0002-2944-1067.

Correspondencia a:carlos.r.puerto.s@gmail.com

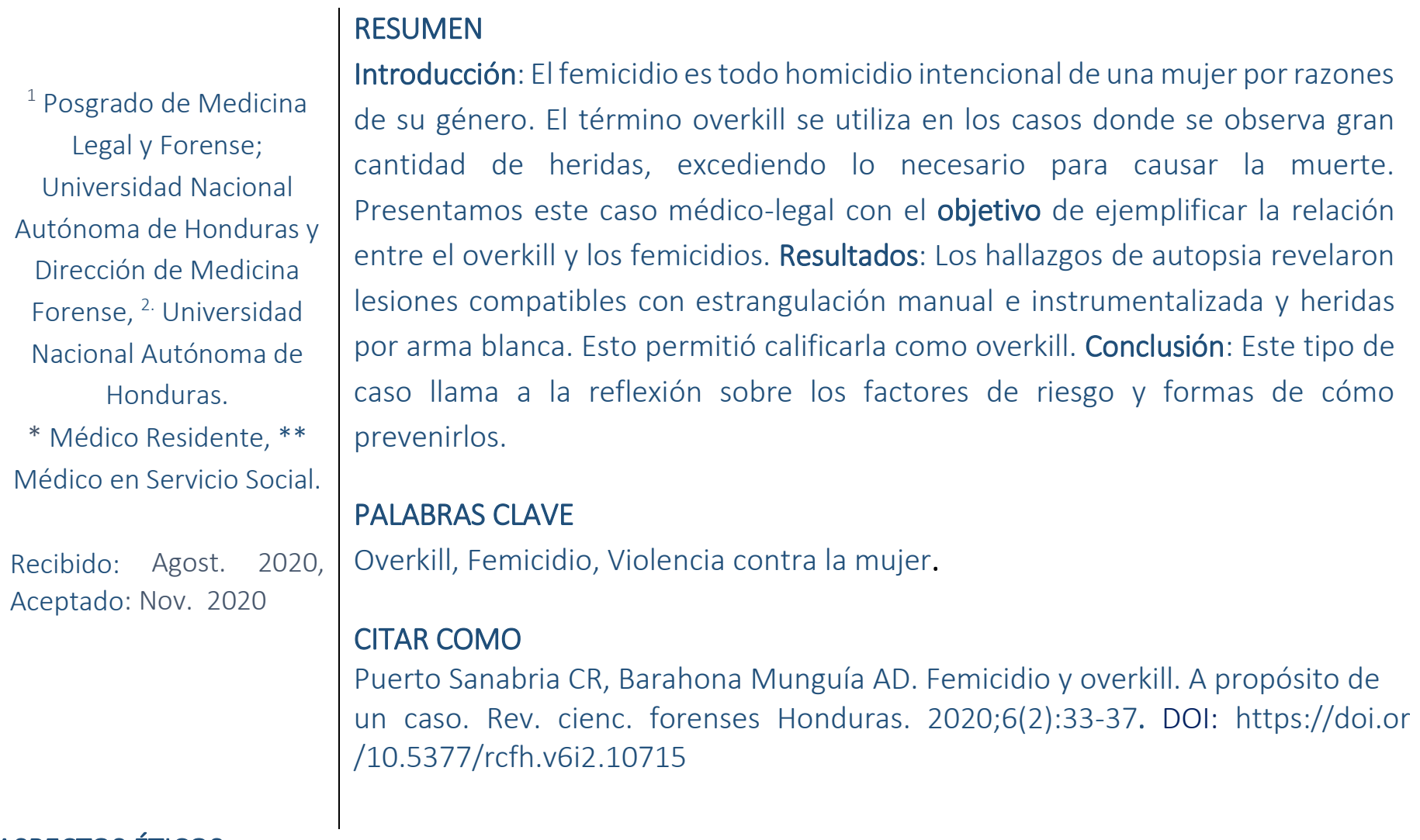

\section{ASPECTOS ÉTICOS}

strangulation and stab wounds; this allows it to be

Los autores declaran que no hubo conflicto de interés en la preparación de este artículo.

\section{ABSTRACT \\ Introduction: Femicide is any gender-based intentional homicide of a woman. The term overkill is used in cases where a large number of wounds is observed, exceeding what is necessary to cause death. We present this medico-legal case with the objective of exemplifying the relationship between overkill and femicides. Result: The autopsy findings were compatible with manual and instrumental} classified as overkill. Conclusion: This type of case call us about risk factors and to reflect on ways to prevent them.

\section{KEYWORDS}

Overkill, Femicide, Violence against women.

\section{INTRODUCCIÓN}

La violencia tiene gran importancia en el sistema social, de salud y de justicia. En el 2003, la OMS la declaró como un problema de salud pública 
prevenible ${ }^{1,2}$. La violencia de género es toda violencia dirigida contra una persona por motivos de su género; la violencia intrafamiliar y la violencia de pareja íntima son algunos de sus exponentes. La OMS definió la violencia contra la mujer (VCM) en la Convención de Belem Do Para como "cualquier acción o conducta, basada en su género, que cause muerte, daño o sufrimiento físico, sexual o psicológico a la mujer, tanto en el ámbito público como en el privado". Basándonos en el concepto de las Naciones Unidas, la VCM se define como toda acción o amenaza que cause o pueda causar daño, con la intención de limitar la independencia y autonomía de la mujer, sea de manera pública o privada, incluyendo la violencia letal ${ }^{2-8}$.

En 1976, Diana Russel utilizó por primera vez el término femicidio, sin embargo, su propagación se generalizó hasta años recientes. Se entiende por femicidio todo homicidio intencional de una mujer por razones de su género, con odio y desprecio por su condición de mujer, siendo la manifestación más extrema de la VCM. Por lo tanto, no todos los homicidios de mujeres son femicidios, los cuales se diferencian de los otros homicidios por: la motivación de la conducta homicida, características del agresor, relación víctima-victimario, factores de riesgo y consecuencias. Una característica típica de los femicidios es que suele existir una relación íntima previa entre la víctima y el perpetrador, usualmente un hombre conocido y cercano, o una situación en la cual el perpetrador conoce a la víctima porque la ha observado a la distancia. Estos son conocidos como femicidios íntimos. Los femicidios varían en mecanismo e instrumento de muerte dependiendo de la disponibilidad de armas, contexto sociopolítico e influencias culturales, que incluyen relaciones familiares, actividad criminal, uso de drogas y moralidad 2,4,5,7,9-14.

El término overkill, o "rematar", se utiliza para definir patrones donde se observan gran número de heridas excediendo lo necesario para causar la muerte. También se ha utilizado para señalar que ha habido dos o más mecanismos involucrados en la muerte. A pesar de ser un término aceptado en la semiología de la medicina legal, hay poca literatura donde se le relaciona con los femicidios. Es por esta razón que presentamos este caso médico-legal con el objetivo de ejemplificar la relación entre el overkill y los femicidios 2,4,13,15.

\section{Presentación de caso}

Historia Médico Legal: mujer en unión libre, cursando la segunda década de la vida, en un entorno de pobreza. Antecedentes de haber interpuesto múltiples denuncias por violencia doméstica por su compañero de hogar. Los vecinos reportaron gritos provenientes de la casa en la madrugada. A la mañana siguiente, su cuerpo se encontró en el suelo de su casa en un lago hemático. Según reporte de investigación, la víctima se encontraba separada de su pareja hacía dos meses.

Hallazgos de la autopsia: en el examen externo se encontró: petequias y cianosis central en cara; lesiones patrón compatibles con estrangulación manual, presencia de un surco incompleto compatible con estrangulación instrumentalizada, y heridas por arma blanca punzocortante en cuello; múltiples hematomas y equimosis de diferente data dispersos en todo el cuerpo. Al examen interno se encontró: laceración de la vena yugular derecha y palidez orgánica generalizada, como se muestra en la Figura 1.

\section{Discusión}

La mayoría de los casos de violencia de pareja íntima (VPI) no terminan en homicidio pues prevalece la violencia no letal contra las mujeres. Sin embargo, el antecedente de VPI es el factor de riesgo más importante para el femicidio. El perpetrador es un hombre conocido, generalmente 


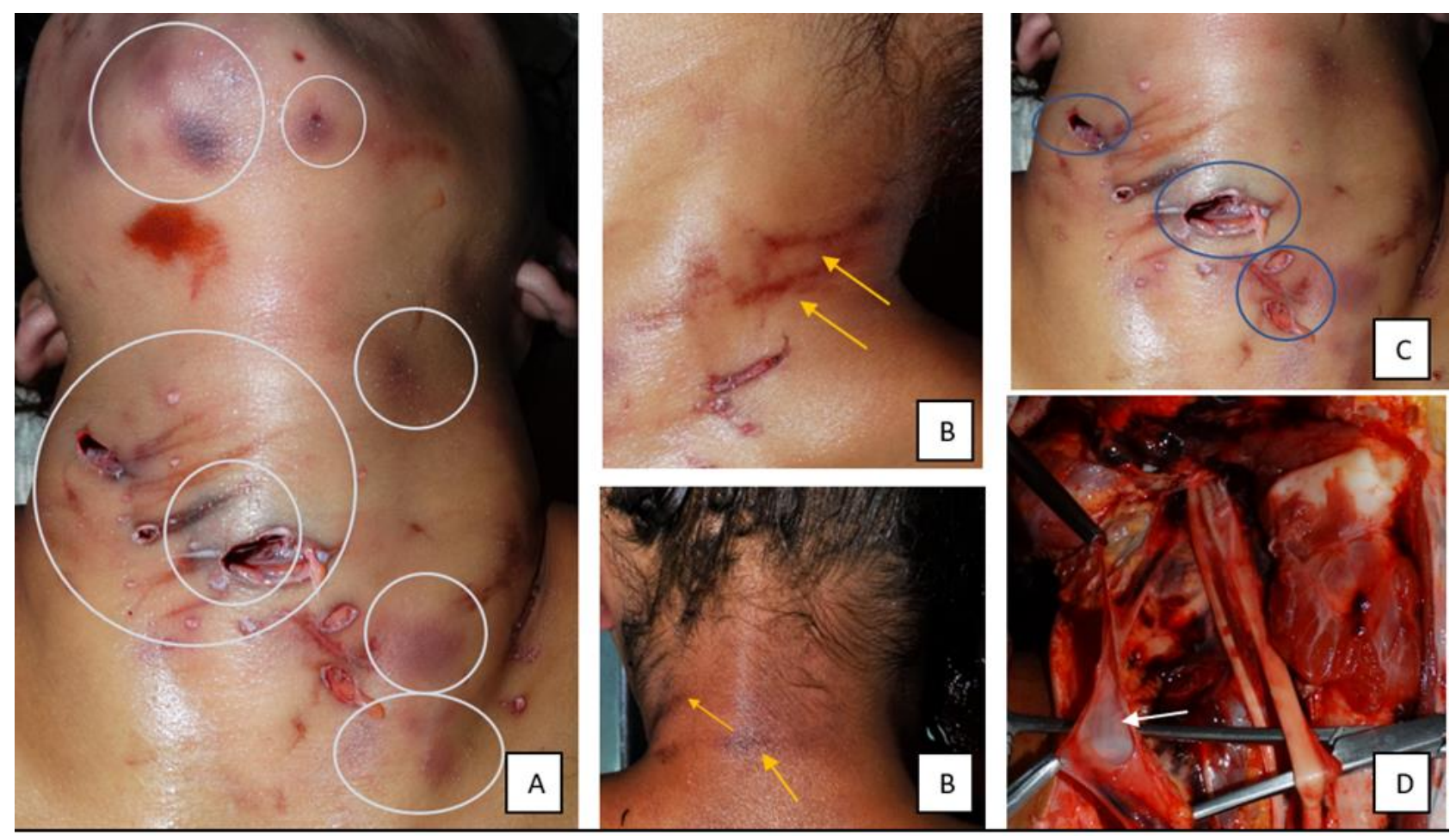

FIGURA 1. A. Círculos blancos: equimosis rojo-negruzcas localizadas en la región inframandibular, región lateral izquierda y anterior del cuello, lesiones patrón compatibles con las producidas por estrangulación manual. B. Flecha amarillas: dos surcos horizontales, no apergaminados, incompletos, compatibles con los producidos por estrangulación instrumental. C. Círculos azules: Heridas por arma blanca punzocortantes. D. Flecha blanca: Laceración de la vena yugular interna derecha

una pareja íntima (esposo/ compañero actual o anterior), como en el presente caso. En el 2018, el Instituto Universitario en Democracia, Paz y Seguridad informó que en Honduras el agresor fue la pareja en el $79.5 \%$ de los casos, la ex pareja o el pretendiente de la víctima, en el $17.9 \%$. Se calcula que los homicidios de parejas íntimas (HPI) o femicidio íntimo representan el 14\% del total de todos los homicidios y el $38 \%$ de los homicidios de mujeres 4,7,11,14,16

El femicidio íntimo, es el desenlace de una historia previa y recurrente de VPI y es el tipo de homicidio doméstico más común, representando el $70 \%$ de los casos. Las causas más prevalentes de este fenómeno son: amenazas de separación o el término de la misma, consumo de alcohol y celopatía. El periodo inmediato a la separación es el más peligroso, particularmente los dos a tres meses después de la separación, hecho que se constata en este caso. Muchos factores tienen un papel en los femicidios incluyendo los relacionados con la víctima, el perpetrador y las dinámicas previas de la pareja ${ }^{1,2,4,6,10}$, como se muestra en el Cuadro 1.

Las causas de muerte más frecuentes de femicidio íntimo son las heridas por arma de fuego, seguido por arma blanca o punzocortante (muchas de ellas con patrón de overkill) y la estrangulación/ sofocación. Según Vergel, un 46-60\% de femicidios íntimos están asociados a overkill, el cual está también estrechamente relacionado con víctimas jóvenes, en edad reproductiva, como es el caso en discusión. Debido a su ambiente predominantemente doméstico, las armas utilizadas suelen ser aquellas fácilmente encontradas en el hogar. Las heridas por VPI y HPI 
Cuadro 1. Factores Asociados al Femicidio (Los factores asociados se clasificaron según:

\begin{tabular}{|c|c|c|}
\hline Víctima & Victimario & Dinámica de la Pareja \\
\hline Diferencia de edad significativa & Enfermedad mental & $\begin{array}{l}\text { Historia de ser víctima de cualquier } \\
\text { tipo violencia }\end{array}$ \\
\hline Dependencia de pareja & Acceso a arma de fuego & Múltiples parejas \\
\hline Ama de casa & Consumo o abuso de sustancias & Pobreza absoluta \\
\hline Abusos durante el embarazo & Comportamiento controlador & Analfabetismo \\
\hline Separación & Desempleo & Entorno machista \\
\hline Tener hijos de relación previa & Ingresos bajos & Estado conyugal \\
\hline
\end{tabular}

víctima, victimario y dinámica de la pareja) ${ }^{1,2,4,6,7,9,16}$.

suelen encontrarse en cabeza, cuello y cara, particularmente heridas en el tercio medio de la cara o maxilofaciales $1,2,4,7,9,10,17$.

El overkill es fácil de apreciar cuando las heridas son abundantes. Las heridas suelen localizarse en cuello y región precordial del tórax. Este patrón se ha relacionado con heridas por arma punzocortante. En el presente caso los hallazgos en la autopsia, son compatibles con tres tipos de traumatismo que pudieron ocasionar la muerte: estrangulación manual, instrumental y herida por arma blanca; esto nos permite calificar el caso como overkill. La causa de muerte y el análisis de la escena del crimen denotan odio y desprecio para el género por parte del victimario, condiciones necesarias para tipificar el homicidio como femicidio. 2,4,15.

\section{CONCLUSIONES}

La violencia contra la mujer es un problema global. Los femicidios íntimos se asocian a violencia extrema con diferentes tipos de mecanismos capaces de causar muerte, lo que define el overkill. Este tipo de caso nos debe llamar a la reflexión, para identificar señales previas en la dinámica de la pareja con el fin de diseñar estrategias para prevenirlos.

\section{REFERENCIAS BIBLIOGRÁFICAS}

1. Matias A, Gonçalves M, Soeiro C, Matos M. Intimate partner homicide: A meta-analysis of risk factors. Aggress Violent Behav. 1 de enero de 2020; 50:101358.

2. Guías, Protocolos y Reglamentos - Instituto Nacional de Medicina Legal y Ciencias Forenses [Internet]. 2020 [citado 15 de mayo de 2020]. Disponible en: https://www.medicinalegal.gov.co/normalizacionforense/guias-protocolos-y-reglamentos

2. Sanhueza Morales T. Violencia en las relaciones amorosas y violencia conyugal: Convergencias y divergencias. Reflexiones para un debate. Última 
Década. julio de 2016;24(44):133-67.

4. (PDF) Relación entre el overkill por arma blanca y el femicidio íntimo Relation between Intimate Femicide and Stabbing Overkill Historia del caso [Internet]. ResearchGate. 2020 [citado 15 de mayo de 2020]. Disponible en: https://www.researchgate.net/publication/306057 125 Relacion entre el overkill por arma blanca y el femicidio intimo Relation between Intimat e Femicide and Stabbing Overkill Historia del c $\underline{\text { aso }}$

5. An examination of femicides in Peru between 2009 and 2014. - PubMed - NCBI [Internet]. 2020 [citado 15 de mayo de 2020]. Disponible en: https://www.ncbi.nlm.nih.gov/pubmed/27496802

6. Goussinsky R, Yassour-Borochowitz D. "I killed her, but I never laid a finger on her" - A phenomenological difference between wife-killing and wife-battering. Aggress Violent Behav. 1 de noviembre de 2012;17(6):553-64.

7. Unidad de Género - Instituto Universitario en Democracia, Paz y Seguridad [Internet]. 2020 [citado 15 de mayo de 2020]. Disponible en: https://iudpas.unah.edu.hn/observatorio-de-laviolencia/boletines-del-observatorio-2/unidad-degenero/

8. Convención interamericana para revenir, sancionar y erradicar la violencia contra la mujer «convención de Belem do ara» [Internet]. [citado 11 de octubre de 2020]. Disponible en: https://www.oas.org/juridico/spanish/tratados/a$\underline{61 . h t m l}$

9. Moreschi C, Da Broi U, Zamai V, Palese F. Medico legal and epidemiological aspects of femicide in a judicial district of north eastern Italy. J Forensic Leg Med. 1 de abril de 2016; 39:65-73.
10. Fong $\mathrm{W}-\mathrm{L}$, Pan $\mathrm{C}-\mathrm{H}$, Lee $\mathrm{JC}-\mathrm{I}$, Lee $\mathrm{T}-\mathrm{T}$, Hwa H-L. Adult femicide victims in forensic autopsy in Taiwan: A 10-year retrospective study. Forensic Sci Int. septiembre de 2016; 266:80-5.

11. Salameh HOG, Salameh RJ, Shwaiki M, AbderRahman H. Forensic medical aspects of femicide in Jordan. J Forensic Leg Med. 1 de mayo de 2018; 56:90-3.

12. Femicidio en Honduras Publications [Internet]. 2020 [citado 15 de mayo de 2020]. Disponible en: https://publications.iadb.org/publications/spanish/ document/Femicidio en Honduras es es.pdf

13. García J, Franco JA. El feminicidio en Bogotá, una mirada desde el abordaje médico-legal. Cuad Med Forense. 2018;24(1-2):27-34.

14. Zaghloul NM, Megahed HM. A descriptive medico-legal study of female deaths in cairo governorate, Egypt. J Forensic Leg Med. 1 de agosto de 2019; 66:25-32.

15. Solarino B, Punzi G, Di Vella G, Carabellese F, Catanesi R. A multidisciplinary approach in overkill: Analysis of 13 cases and review of the literature. Forensic Sci Int. 1 de mayo de 2019; 298:402-7.

16. Incidence of female homicide in the Transkei sub-region of South Africa (1993-2015). - PubMed NCBI [Internet]. 2020 [citado 15 de mayo de 2020]. Disponible en: https://www.ncbi.nlm.nih.gov/pubmed/29571166

17. Vergel J, Trompetero-González A-C. The magnitude of the injury pattern in femicides by stabbing in Colombian women. Rev Fac Med. 1 de octubre de 2017; 65(4):559-63. 